\title{
LECCIONES EPISTEMOLÓGICAS DE LA HISTORIA DE LA GEOMETRÍA
}

\author{
Carlos Arturo Londoño Ramos \\ Universidad Pedagógica y Tecnológica de Colombia \\ Blanca Inés Prada Márquez \\ Universidad Industrial de Santander \\ ...no podemos por menos de reconocer la verdad de aquel principio de Platón \\ según el cual todo aquel que desee con éxito dedicarse a la metafísica debe \\ prepararse para ello con el estudio de la geometría. \\ Johann Heinrich Pestalozzi. Cartas sobre la Educación infantil.
}

\section{Resumen}

El recorrido histórico sobre la creación de las geometrías no euclidianas, es decisivo para juzgar los criterios que la epistemología ha planteado en el problema de la validez de los enunciados de las ciencias formales, así como con respecto al interrogante por los razonamientos implicados en estas ciencias. La creatividad manifiesta en las geometrías alternativas, no-euclidianas, tiene repercusiones en la filosofía pues con ellas se muestra que para la elaboración de teoremas se requiere una multiplicidad de formas de los enunciados, que no se puede reducir simplemente a los enunciados analíticos, sino que son verdaderas construcciones, en consecuencia, también cambian los puntos de vista pedagógicos.

Palabras clave: epistemología de la geometría, geometrías no euclidianas, filosofía de las ciencias formales.

\footnotetext{
* Correos-e: londonocarlosarturo@hotmail.com.pradamblancaines@gmail.com
} 


\begin{abstract}
The historical background of the creation of non-Euclidean geometries, is crucial for judging the criteria that epistemology has raised with regard to the question of validity of statements of the formal sciences as well, as with the question of the reasoning involved in these sciences. The creativity manifested in non-Euclidean alternative geometries, has implications for philosophy because with these it shows that the development of theorems requires a multiplicity of statement forms, which cannot be simply reduced to analytical statements, but which are true constructions, therefore changing pedagogical viewpoints.
\end{abstract}

Key words: epistemology of geometry, non-Euclidean geometries, philosophy of the formal sciences.

\title{
Introducción
}

Desde la antigua Grecia, la matemática ha suscitado problemas filosóficos, e incluso en la escuela platónica la geometría se convirtió en uno de los modelos ejemplares para las otras ciencias. La filosofía de las ciencias lógicomatemáticas se pregunta en qué radica la validez de sus enunciados. ¿Por qué aceptamos sus enunciados como universales? ¿En razón de qué aprobamos sus enunciados como verdaderos a priori, es decir, sin tener en cuenta la experiencia? Cuál es la característica especial de los conceptos matemáticos? Por ejemplo, el punto, que según Euclides se define como "lo que no tiene partes" no es un espacio real, sino conceptual. En la filosofía también se ha contado con problemas que no son estrictamente lógico-matemáticos, pero que inciden en su comprensión y en la explicación de su génesis, tales como las relaciones entre la validez de estas ciencias formales con su origen, es decir, la conexión con el desarrollo histórico y con el aprendizaje individual, tal como lo investiga Jean Piaget. Este problema, a su vez, está enlazado con otro, ¿si las ciencias lógico-matemáticas sólo tratan con conceptos, principios lógicos y deducciones, por qué son aplicables, en las ciencias naturales y en la tecnología? A este problema se acercan algunas corrientes de interpretación denominadas "intuicionistas" y la sicología cognitiva. 
La filosofía de la matemática se complicó aún más cuando aparecieron las geometrías no euclidianas, pues a partir de ellas comienzan a existir diferentes sistemas formales igualmente válidos, lo cual reactiva la pregunta tanto por el "ser" de la geometría como por la especificidad de su verdad.

Epistemólogos de la ciencia como Imre Lakatos, Gaston Bachelard y Piaget insisten en la importancia de integrar la enseñanza de las ciencias con la filosofía y la historia de su desarrollo, integración indispensable para entender el dinamismo y la evolución del conocimiento, puesto que el pensamiento científico se ha ido desarrollando y perfeccionando poco a poco, gracias al trabajo investigativo, crítico y constructivo. Los autores señalados insisten también en la importancia de la reflexión epistemológica para la comprensión de la estructura interna de las teorías científicas, señalando además que la historia de la ciencia ayuda a comprender no sólo el dinamismo de las ciencias, sino también sus fundamentos cuando dicha historia no se reduce a una simple relación de hechos, sino que se convierte en historia juzgada, en una reconstrucción racional del proceso mismo del saber científico, de ahí que diga Lakatos, parafraseando a Kant:"la filosofía de la ciencia sin la historia de la ciencia es vacía; la historia de la ciencia sin la filosofía de la ciencia es ciega". Por su parte, para Bachelard: "Asir el pensamiento científico contemporáneo en su dialéctica y mostrar su novedad esencial es el propósito filosófico..."2.

\section{Antecedentes de la geometría de Euclides}

El pensamiento matemático aplicado en la solución de los problemas prácticos de la vida va a encontrar en los griegos una dimensión muchos más universal y abstracta. En relación con las matemáticas, su desarrollo empieza a manifestarse con Tales (624-545/6 a J.C.) y con Pitágoras (580-500 a J.C.) quienes vivieron en las costas jónicas. Por su situación geográfica viajaban fácilmente a Mesopotamia y Egipto, y allí recibieron de primera mano los aportes matemáticos y astronómicos de estas dos civilizaciones, dándoles un desarrollo original y teórico.

1 LAKATOS, Inre. Historia de la ciencia y sus reconstrucciones racionales. Madrid: Tecnos. 1887, p 11.

2 BACHELARD, G. El nuevo espíritu científico. México: Nueva Imagen, 1985, p. 20. 
Poco se sabe de la vida de Tales de Mileto, la tradición le atribuye la predicción del eclipse del año 585 a. C., lo califica además de primer filósofo, colocándolo entre la lista de los siete sabios del mundo antiguo y es considerado además como un hombre particularmente inteligente, el primer matemático auténtico, es decir, como el padre de la organización deductiva de la geometría, atribuyéndole en primer lugar la proposición que hoy se conoce como el teorema de Tales: "Un ángulo inscrito en una semicircunferencia es un ángulo recto", del cual dicha tradición, ofreció algunas demostraciones. También se le atribuyen otros seis teoremas sobre los cuales habría ofrecido alguna demostración. ${ }^{3}$ De otra parte, se le atribuye el primer esfuerzo de cálculo para medir la altura de las pirámides de Egipto, como también el planteamiento de un problema para hallar, en un momento determinado, la distancia que separa un barco de la costa.

Pitágoras fue el fundador de la escuela que lleva su nombre; su vida está plagada de misterio, envuelta por la leyenda y por una especie de culto, casi religioso. Viajaría a Egipto y Babilonia, y posiblemente, también a la India, viajes en los cuales, no sólo asimiló conocimientos matemáticos y astronómicos sino también religiosos, siendo casi contemporáneo de Buda, Confucio y Lao-Tse.

Después de sus viajes se estableció en Crotona, en la costa sudeste donde hoy queda Italia, pero en aquella época se conocía como la Magna Grecia, allí fundó su escuela o su secta. La asociación pitagórica, cofradía o hermandad, estaba basada en la comunidad de bienes y de descubrimientos. Su principal objetivo era la purificación del alma o catarsis, cultivando el arte de la música y la ciencia de las matemáticas, siguiendo el camino de la filosofía, palabra que desde entonces ha significado amor a la sabiduría; tenían también un gran sentido de fraternidad y cultivo de la amistad. ${ }^{4}$ La escuela pitagórica se caracterizó, entre otras cosas, por la búsqueda en el mundo inteligible del principio generador del universo, introduciendo la novedad de que en el fondo del mundo material el número es lo único accesible al intelecto. ${ }^{5} \mathrm{El}$ lema de la escuela pitagórica habría sido "todo es número" y su contribución más importante a la geometría sería su famoso teorema.

3 CID, Felip. Historia de la ciencia. Barcelona: Planeta, tomo I, 1977, p. 67.

4 VERA, Francisco (Comp.). Científicos griegos. Madrid: Aguilar, Tomo I. , 1970, p. 54.

5 BOYER, Carl. Historia de las matemáticas. Madrid: Alianza. 1999, p. 78. 
El teorema de Pitágoras dice que en todo triangulo rectángulo el cuadrado de la hipotenusa es igual a la suma de los cuadrados de los catetos. Aunque el teorema pueda proceder de los babilonios, fueron los pitagóricos los primeros en darle una demostración. El famoso teorema dio origen al descubrimiento del número irracional cuando aplicaron el teorema al triángulo rectángulo isósceles de catetos iguales a la unidad, resultando lo que algunos estudiosos del pitagorismo, entre otros Paul Tannery, han llamado "el escándalo de la matemática pitagórica".

Los pitagóricos consideraron la matemática como la ciencia suprema, la cienciatipo del conocimiento: todo su sistema tiende al matematismo. El matematismo cuasi pitagórico ha impregnado todo el desarrollo de la ciencia moderna desde Galileo, tendencia que se sintió mucho más fuerte en el siglo XX como bien lo señala Bertrand Russell al decir que "lo más extraño de la ciencia contemporánea sea quizá, su vuelta al pitagorismo" 6 .

\section{La geometría de Euclides, monumento intelectual difícil de derrumbar}

Euclides de Alejandría enseñó matemáticas durante el reinado de Ptolomeo I (siglo III a. de J.C.). Hasta nuestros días han sobrevivido cinco obras: Elementos, Los datos, La división de figuras, Los fenómenos y La óptica. La última de estas obras tiene el interés de ser una obra sobre perspectiva o la geometría de la visión directa. Según Boyer los antiguos habían dividido el estudio de los fenómenos ópticos en tres partes: Óptica o la geometría de la visión directa; Catóptrica o la geometría de los rayos reflejados; y Diotrópica o la geometría de los rayos refractados.

La geometría clásica bajo la forma que le dio Euclides dentro de Elementos, pasó durante casi veinte siglos por un modelo insuperable e inigualable de teoría deductiva. Los términos propios de la teoría no son jamás introducidos sin ser definidos. Las proposiciones no son jamás avanzadas sin ser demostradas con excepción de un pequeño número de ellas que son enunciadas a título de principio: la demostración no puede remontarse al infinito y, debe, por lo tanto, reposar sobre algunas proposiciones primeras que han sido escogidas con tal cuidado para que ninguna duda subsista sobre ellas.

6 VERA, Francisco (Comp.). Científicos griegos. Op. Cit,, p. 58. 
Los griegos han razonado con toda la precisión posible dentro de las matemáticas, y dejaron al género humano modelos del arte de demostrar. La geometría dejó con ellos de ser una recolección de recetas prácticas, de enunciados empíricos o concretos, para convertirse en una ciencia racional y formal. De aquí el papel pedagógico privilegiado que siempre se les ha reconocido. Si se hace estudiar esta geometría a los niños no es tanto para darles verdades, sino más bien, para disciplinar el espíritu, ya que su práctica desarrolla habilidades y hábitos para el razonamiento riguroso, como bien lo señala Blanché ${ }^{7}$ cuando dice: "Euclides ha sido, para numerosas generaciones que se han nutrido con su sistema, más que un profesor de geometría, un profesor de lógica. De ahí también que la expresión more geométrico se suela entender como more lógico".

Los elementos están divididos en trece libros o capítulos de los cuales, la primera media docena son sobre geometría plana elemental, los tres siguientes sobre teoría de número y el libro $\mathrm{X}$ sobre los inconmensurables y los tres últimos sobre geometría de sólidos. Sin preámbulo ni prólogo, el libro empieza con una lista de 23 definiciones, las cuales describen los entes geométricos: puntos, líneas, superficies; como ejemplos tenemos las definiciones: "Una figura es lo que se halla contenido por cualquier límite o límites", "Una línea recta es una línea que se extiende plana con los puntos sobre sí misma". Algunas de las definiciones han recibido muchos cuestionamientos posteriores, pero otras permanecen. A continuación de las definiciones vienen cinco postulados, que son enunciados necesariamente supuestos para la deducción geométrica pero que no son demostrables, más bien están destinados a legitimar ciertas construcciones, tal es el caso del segundo postulado: "Toda línea recta finita puede extenderse continuamente en línea recta." El quinto postulado -el famoso talón de Aquiles- de la geometría griega es llamado por antonomasia "postulado de Euclides", que ha hecho gastar en comentarios e intentos de demostración montañas de papel y mares de tinta.

A continuación de los postulados, siguen cinco axiomas que son principios iniciales (o "nociones comunes") o los enunciados más universales, que también pueden ser principios de la lógica o de la aritmética. Aristóteles había hecho una distinción entre axiomas y postulados; los primeros, según él, deben ser

7 BLANCHÉ, Robert. L'axiomatique. París: PUF, 1959, p. 2. 
convincentes por sí mismos, por ser verdades comunes a todas las ciencias, es decir son supuestos necesarios que no requieren demostración, mientras que los segundos son menos evidentes aunque son supuestos necesarios. Esta primera tentativa de axiomatización fundó la geometría sobre bases consideradas indiscutibles durante muchos siglos.

Según señala el filósofo y matemático austriaco Richard Misses en su artículo "Los postulados de la matemática y el entendimiento humano"8 en nuestro aprendizaje preuniversitario se suelen enseñar axiomas de la geometría y de la aritmética como verdades irrefutables, por ejemplo: toda cantidad es igual a sí misma; el todo es mayor que cualquiera de sus partes; todos los ángulos rectos son iguales entre sí, afirmándose de estos axiomas que son verdades autoevidentes y que todos los teoremas matemáticos se siguen de ellos de un modo estrictamente lógico. Investigaciones modernas sobre los fundamentos de la geometría han mostrado que no puede construirse geometría alguna a partir de las escasas proposiciones básicas formuladas como axiomas en los textos didácticos.

Euclides, como Aristóteles con la lógica formal, y posteriormente Pascal, Leibniz y Newton con la física clásica, expresó el ideal de organización axiomática de una disciplina, "ideal que grosso modo podía reducirse a la elección de un pequeño número de proposiciones evidentes dentro de aquel ámbito del saber y a la posterior deducción de todas las demás proposiciones verdaderas en dicho ámbito, partiendo de aquellas"9.

\section{El quinto postulado: talón de Aquiles de la geometría euclidiana}

Desde la época antigua, el quinto postulado de Euclides no había resultado para los estudiosos demasiado evidente. El famoso postulado a veces denominado "de las paralelas" fue formulado así por Euclides: "Si una recta al incidir sobre dos rectas hace los ángulos internos del mismo menores que dos rectos, las dos rectas prolongadas indefinidamente se encontrarán en el lado en que están los (ángulos) menores que dos rectos" (Euclides, Libro I, Postulado 5) ${ }^{10}$. (figura 1)

8 NEWMAN, John Von Sigma. El mundo de las matemáticas. Barcelona: Grijalbo, tomos IV y V, 1983.

9 REALE Giovanni y ANTISERI, Darío Historia del pensamiento filosófico y científico. Barcelona: Herder, Tomo III, 1988, p. 328.

${ }^{10}$ EUCLIDES. Elementos. Trad. María Luisa Puertas. Madrid: Gredos, 1991, p. 197. 


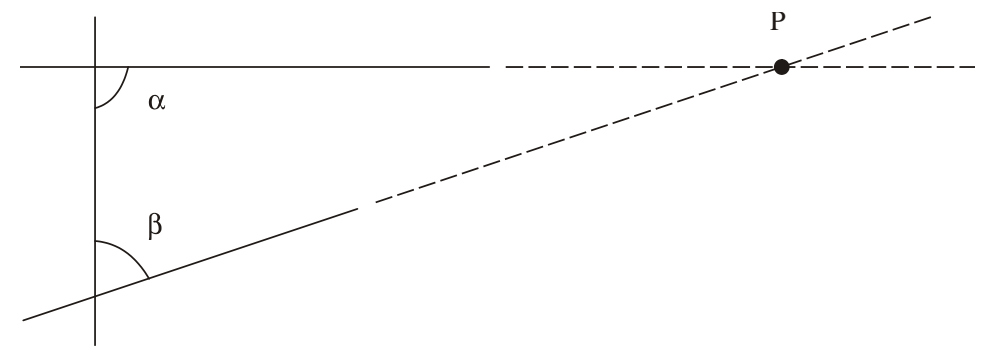

Figura 1. Postulado de las paralelas

Como bien lo explican Antiseri y Reale, este postulado nos indica que, si tenemos en un plano una recta $\mathbf{S}$ y un punto $\boldsymbol{P}$ exterior a ella, en el plano sólo existe una recta $\mathbf{r}$ que pase por el punto $\boldsymbol{P}$ y sea paralela a la recta $\mathbf{s}$, en el sentido de que jamás la encuentre. Sucede tal cosa, continúan diciendo los autores señalados, cuando la recta $\mathbf{r}$ y la recta $\mathbf{s}$ se encuentran con la recta $\mathbf{t} \mathbf{y}$ forman dos ángulos rectos (como en la figura 2a) o dos ángulos cuya suma sea igual a dos rectos (como en la figura $2 \mathrm{~b}$ ).

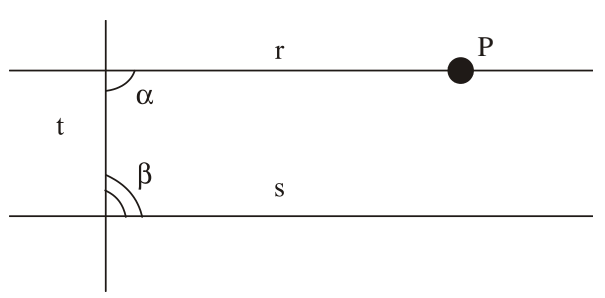

Figura 2a. Primera interpretación del postulado de las paralelas

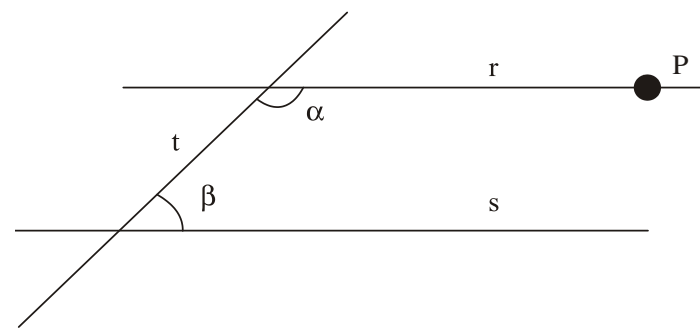

Figura 2b. Segunda interpretación del postulado de las paralelas

El problema está en que esta proposición no es algo evidente, y en otro modelo puede resultar falsa. En efecto, si el plano que contiene la recta s y el punto $\mathbf{P}$ exterior a ella queda limitado a la zona interior del círculo, entonces se aprecia de inmediato (como se intuye a través de la figura 3) que hay muchas rectas que pasan por $\boldsymbol{P}$ y que no se encuentran con $\mathbf{S}$. Si aumentamos el radio del círculo, disminuirá la cantidad de rectas que pasan por $\mathbf{P}$ y no se interseccan. ¿Qué intuición, qué autoevidencia podrá garantizarnos que esta situación dejara de darse cuando el plano sea limitado?, se pregunta Agazzi ${ }^{11}$.

${ }^{11}$ Cfr. AGAZZI Evandro y PALADINO, D. Le geometrie non - euclidienne e i fondamenti della geometria. Milán, Mondarori, 1978. 


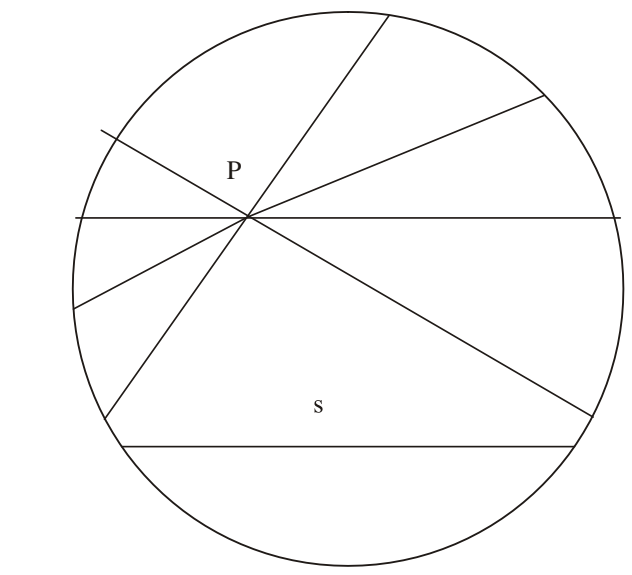

Figura 3. Tercera interpretación del postulado de las paralelas

Siguiendo las explicaciones muy didácticas que dan Reale y Antiseri se podría decir que si hay una recta $\mathbf{s}$ en un plano, y un punto $\mathbf{P}$ exterior a ella y por el que pasa la recta $\mathbf{r}$ que se encuentra con la recta $\mathbf{S}$ en el punto $\boldsymbol{A}$, con el propósito de que la recta $\mathbf{r}$ se convierta en paralela a la recta $\mathbf{s}$, si se hace girar en el sentido contrario a las agujas del reloj, la recta r que se encontrará sucesivamente con la recta $\mathbf{s}$ en los puntos $\boldsymbol{B}, \boldsymbol{C}, \boldsymbol{D}, \boldsymbol{E}$, estos se alejarán cada vez del punto $A$, pero si se trata de un plano infinito ¿Cómo podrá controlarse dicha separación? ¿Es posible afirmar que dos rectas paralelas se encuentran en el infinito? (figura 4)

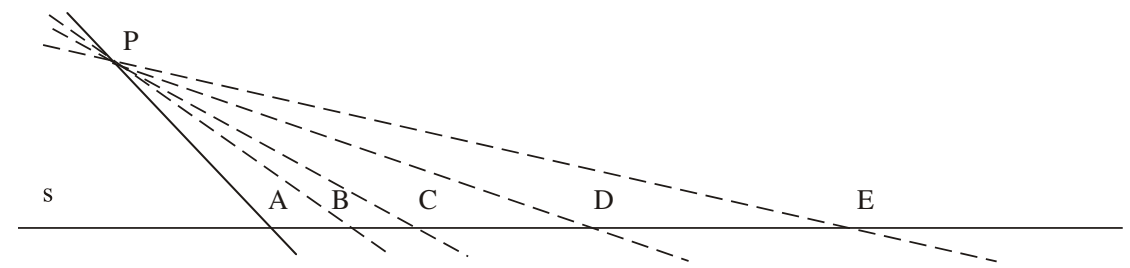

Figura 4. Cuarta interpretación del postulado de las paralelas

Las anteriores consideraciones muestran algunas de las muchas perplejidades que acompañaron la reflexión en torno al quinto postulado de Euclides, a lo largo de más de 20 siglos de aceptación, como la única geometría verdadera. 


\section{Desarrollo de las geometrías no euclidianas}

A lo largo de la historia del pensamiento griego, árabe, y renacentista, los matemáticos y filósofos estuvieron siempre muy inquietos frente al quinto postulado, al que veían más como un teorema que como un postulado, de ahí los intentos por demostrarlo como si fuera una consecuencia de los primeros cuatro que parecían sencillos y evidentes. Sin embargo, todos los intentos fracasaron.

En el siglo XVIII el jesuita Girolamo Saccheri (1677-1733), en su obra Euclides enmendado de todos sus defectos (1733) se propuso negar el postulado euclidiano de la paralela y dedujo naturalmente todas las consecuencias lógicas de tal negación, tratando de buscar una contradicción que demostrara, mediante el absurdo, el famoso postulado. ${ }^{12}$ Algunos estudiosos de la matemática, piensan que éste podría ser un primer esfuerzo coherente por construir una geometría no euclidiana. Otros matemáticos como Lambert, Taurinus, Wachter y Reid, ofrecieron también sus aportes en este esfuerzo por demostrar o negar el quinto postulado, sin embargo, todos ellos lo que intentaban era, o bien negar la geometría euclidiana creando una nueva geometría, o bien mostrar que definitivamente la geometría de Euclides era la única geometría posible, por lo tanto, no consideraron nunca la posibilidad de que pudieran existir dos o más geometrías igualmente válidas. Este honor sólo lo logran Gauss (17771855), Bolyai (1802-1860) y Lobachevsky (1793-1856), quienes van a mostrar la posibilidad de aceptar dos o más sistemas de proposiciones opuestas como simultáneamente verdaderas desde el punto de vista formal, atribuyendo a los mundos geométricos que les correspondan el mismo valor ontológico, la misma "realidad racional" o inteligible y, conservando, además, la misma interpretación semántica para los términos fundamentales como línea, recta, distancia, longitud, congruencia, etc., en los diferentes sistemas de axiomas

Karl Friedrich Gauss, profesor en la Universidad de Göttingen, vio con toda claridad, a principios del siglo XIX, la no demostrabilidad del quinto postulado de Euclides y la posibilidad de construir sistemas geométricos distintos del euclidiano, pero dice, no publicó sus investigaciones por temor al "griterío de los torpes", valiéndose de una frase ya expresada por Kepler cuando explicaba

${ }^{12}$ Cfr. AGAZZI, Evandro y PALADINO, D. (1978). Le geometrie non-euclidienne e $i$ fondamenti della geometria. Milán, Mondarori, 1978. 
su temor de publicar sus ideas en relación con la aceptación del sistema copernicano.

Gauss anotaba sus descubrimientos en un diario. El famoso Diario permaneció oculto hasta 1901 cuando la Real Sociedad Científica de Gontinga, para celebrar el sesquicentenario de su fundación, logró que el nieto de Gauss le cediera el precioso documento y se mostró así a la luz pública su extraordinario ingenio. Entre sus 146 resultados estaba la no demostrabilidad del quinto postulado de Euclides y la posibilidad de construir sistemas geométricos distintos del euclidiano. El lema de Gauss era: pauca sed matura (poco pero maduro); en efecto, su mente estaba tan rebosante de ideas que nunca tuvo el tiempo suficiente para organizarlas y darles la perfección que deseaba tuvieran, antes de publicarlas, no obstante, vivía continuamente preocupado por este problema; en carta a F.A. Taurinos, refiriéndose a la geometría no-euclidiana, le escribía:

Lo que en este sistema repugna a nuestra razón es que si éste fuera verdadero, en el espacio existiría un segmento (geométricamente) definido si bien desconocido por nosotros. Sin embargo, me parece que si prescindimos de la sabiduría verbal de los metafísicos vacía de significado, sabemos muy poco o casi nada de la esencia del espacio; no podemos confundir lo que a nuestros ojos resulta algo no natural con lo absolutamente imposible ${ }^{13}$.

Dado que Gauss no publicó sus consideraciones sobre el quinto postulado la gloria de la fundación de la geometría no euclidiana corresponde al ruso Nicolai Ivanovitch Lobachevsky, profesor de la Universidad de Kazan, quien es considerado hoy el "Copérnico de la geometría", el hombre que revolucionó este campo, creando en él una rama totalmente nueva, la geometría lobachevskiana, con la cual logró demostrar que la geometría de Euclides no era la ciencia exacta de la verdad absoluta, como se había admitido hasta entonces. Con la publicación del artículo en un periódico "Sobre los principios de la geometría" (1829) y la obra Nuevos principios de la geometría con una teoría completa de las paralelas (1835) da nacimiento oficial a las geometrías no euclidianas, en cuyo trabajo logró realizar la revolucionaria tarea de hacer pública una geometría construida expresamente sobre una hipótesis que

${ }^{13}$ LONBARDO-RICE. Lobacevskij. Nouvi princípi della geometria conme teoria completa delle parallele. Universale Scientifica Boringhieri. p. 13-54. Citado por USINI, Sonia. "La aportación de Gauss a la geometría hiperbólica: su carteo con matemáticos y científicos de la época”. En: Miscelánea matemática. N 33, Cinvestav, SMM, (2001), p. 1-19. 
contradecía el quinto postulado de Euclides. Con el nuevo planteamiento logra Lobachevsky construir una geometría armónica, pero al mismo tiempo, opuesta al sentido común, por lo cual él mismo llamó a su obra Geometría imaginaria (1835). Pero como ha sucedido varias veces en la historia de la ciencia que dos investigadores logran llegar a los mismos resultados simultáneamente, el mismo año Janos Bolyai escribió La ciencia absoluta del espacio con un subtítulo que bien puede hacer alusión a Kant y que dice "Independiente de la verdad o falsedad del XI axioma de Euclides (Nunca podemos decidir a priori)." La pequeña obra es publicada en 1832, como apéndice en la obra Testamento del Wolfgang Bolyai, padre de Janos y amigo de Gauss ${ }^{14}$. La definición (o axioma) 11 dice "El ángulo obtuso es el (ángulo) mayor que un recto"15. Bolyai quería demostrar que se puede hacer triángulos con arcos en los cuales no se cumple la definición de Euclides.

En la geometría de Lobachevsky, se utiliza la línea curva como en una silla de montar; curvaturas que son denominadas "negativas", a diferencia de las líneas que en la geometría de Riemann son curvaturas de la esfera, denominadas "positivas", y en la geometría euclidiana la curvatura es de magnitud 0. En la geometría de Lobachevsky desde un punto exterior a una recta siempre se puede trazar más de una línea paralela a dicha recta. La suma de los ángulos de un triangulo es siempre menor que dos ángulos rectos. Además, la razón de la circunferencia de un círculo a su diámetro es siempre menor que el número .

A pesar de que la obra de Lobachevsky fue publicada en alemán en 1840, la geometría no euclidiana (el término usado por Gauss) continuó durante varios años convertida en un aspecto marginal de la matemática, hasta que logró integrarse completamente en ella, gracias a las concepciones extraordinariamente generales que le aportó el alemán y profesor de la Universidad de Humboldt Georg Friedrich Bernhard Riemann (1826-1866), con la publicación, en 1867, de su tesis de habilitación orientada por Gauss Sobre la hipótesis en que se

${ }^{14} \mathrm{El}$ reconocimiento a la obra de Janos Bolyai fue tardío por el poco apoyo que recibió de Gauss para la edición de su obra, lo cual lo desalentó en su trabajo. En 1896 George Bruce H. en Austin, Texas, con una introducción publica La Ciencia Absoluta del espacio, Ed. Neomon. Y, en 1955, Roberto Bonola publicó en inglés Non-euclidean geometry, que contiene sendas traducciones al inglés de la "Teoría de las paralelas" de Lobachevsky, y de la "Ciencia absoluta del espacio", de Bolyai.

${ }^{15}$ EUCLIDES. Elementos. Trad. María Luisa Puertas. Madrid: Gredos, 1991, p. 193. 
apoyan los fundamentos de la geometría, ${ }^{16}$ que se convertirá en la obra más importante para comprender el nuevo monumento de las geometrías no euclidianas. Nace así el espacio de superficies curvas positivas llamado espacio de Riemann, que fue utilizado más tarde por Einstein en la teoría de la relatividad general, la cual admite que la curvatura del espacio está dada en cada punto y a cada instante por la gravedad.

Riemann generalizó el concepto de curvatura de Gauss extendiéndolo a las tres dimensiones del espacio. En la geometría de Riemann, a diferencia de la de Euclides, se niega que una línea recta pueda prolongarse hasta el infinito dado que es una curva. Existe una longitud máxima hasta la que pueda prolongarse una línea dado que todas son curvas y sobre la esfera la suma de los ángulos de un triangulo es siempre mayor que dos ángulos rectos.

Una explicación bella y didáctica sobre la importancia de la geometría de Riemann nos la dan Einstein e Infeld en su hermosa obra La física aventura del pensamiento. ${ }^{17}$ Allí ellos explican como la geometría de Riemann era noeuclidiana en un sentido mucho más general que en el caso de la de Lobachevsky, donde el problema central era simplemente el de saber cuántas paralelas se podían trazar por un punto exterior a una recta. Según los autores citados, Riemann considera que en un espacio circular, puede pensarse que "su espacio no tenga límites, puesto que uno puede, en una esfera, ir de adelante hacia atrás sin detenerse, sin embargo este espacio sin límites es finito, puesto que se puede dar la vuelta completamente aunque no se le encuentre límite."18

Como bien lo explican Einstein e Infeld, la geometría de Riemann es la geometría esférica extendida a tres dimensiones; para construirla el matemático alemán se vio obligado no sólo a abandonar el quinto postulado de Euclides sino también el primer postulado que dice que "por dos puntos no puede pasar sino una recta", puesto que sobre una esfera, es posible pasar por dos puntos dados sólo un gran círculo, el cual jugará el papel de recta.

De lo expuesto sobre Lobachevsky y Riemann se concluye que por lo menos se pueden encontrar tres geometrías diferentes:

${ }^{16}$ RIEMANN, G.F.B. Riemanniana selecta. Madrid: Ferreirós, 2000.

${ }^{17}$ EINSTEIN - INFELD La física, aventura del pensamiento. Buenos Aires, Losada. 1939.

${ }^{18}$ BOYER, Carl. Historia de las matemáticas. Op. Cit., p. 675. 


\begin{tabular}{|c|c|c|}
\hline $\begin{array}{l}\text { GEOMETRÍA DE } \\
\text { LOBACHEVSKY }\end{array}$ & $\begin{array}{c}\text { GEOMETRÍA DE } \\
\text { EUCLIDES }\end{array}$ & $\begin{array}{c}\text { GEOMETRÍA DE } \\
\text { RIEMANN }\end{array}$ \\
\hline \multicolumn{3}{|c|}{ La suma de los ángulos de un triángulo es: } \\
\hline Menor de dos ángulos rectos & Igual a dos ángulos rectos & Mayor de dos ángulos rectos \\
\hline \multicolumn{3}{|c|}{ El número de paralelas que se pueden pasar sobre una recta dada por un punto dado es: } \\
\hline Infinito & Una & Ninguna \\
\hline \multicolumn{3}{|c|}{ La proporción entre la circunferencia de un círculo y su diámetro es: } \\
\hline Menor que & & Mayor que \\
\hline \multicolumn{3}{|c|}{ El espacio sería: } \\
\hline Hiperbólico & Plano & Elíptico \\
\hline
\end{tabular}

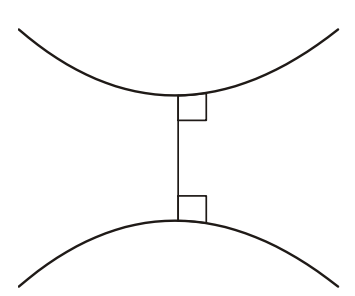

Hyperbolic

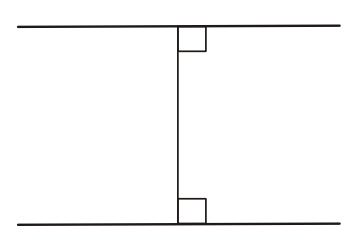

Euclidean

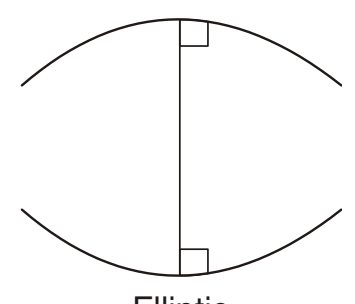

Elliptic

Pero Riemann imaginó otras geometrías, al igual que lo hicieron Hilbert y Veronesse, quienes propusieron geometrías todavía más complicada y extrañas, que fueron llamadas no-arquimedianas. Poincaré mismo imaginó una nueva geometría, uno de cuyos postulados sería que una recta real puede ser perpendicular a ella misma ${ }^{19}$.

\section{Consecuencias epistemológicas}

Leibniz, intentaba sustentar la validez de la aritmética en principios puramente lógicos. Para exponer esta tesis propuso la división de los juicios en verdades de hecho y verdades de razón. Las primeras se sustentan por la vía inductiva de la observación de una multiplicidad de experiencias y, las segundas, por el contrario, son necesarias, como las deducciones de la lógica y de la geometría. También hay formas mixtas como las demostraciones de las leyes de las ciencias físicas que combinan la matemática y la geometría con el experimento. ${ }^{20}$ Leibniz intenta reducir las operaciones aritméticas a definiciones y al principio de identidad, como en el caso de 2 y 2 igual a 4. Para demostrarlo parte de la definición de 2

${ }^{19}$ Cfr. POINCARÉ, Henry La science et l' hypothèse. Paris, Flammarion, 1968.

${ }^{20}$ LEIBNIZ, Gottfried Wilhemlm. Nuevo tratado sobre el entendimiento humano: Del conocimiento. Libro IV. Madrid: Aguilar, 1972, p. 136. 
como constituida por 1 y 1 ; la definición de 3 es 2 y 1 ; la de 4 es de 3 y $1 .{ }^{21}$ No obstante, para Leibniz también existen verdades de razón intuitivas autoevidentes como la de los postulados de la geometría y son aquellas cuya conexión de los conceptos es evidente por sí mismas. Como ejemplo en la física, dice Leibniz: "...que lo es que dos cuerpos no podrán estar en el mismo lugar"22 Este último caso supone la impenetrabilidad de los cuerpos, enunciado que se ha relativizado con la física contemporánea de los agujeros negros.

Hume es más radical que Leibniz. Establece una distinción de conocimientos justificados por la experiencia, que son conocimientos de hecho, a diferencia de los juicios basados en la comparación de ideas según principios lógicos, así sostiene que "cuando una demostración tiene lugar, lo contrario de ella es imposible e implica una contradicción." ${ }^{23}$ La negación de la causalidad, por ejemplo, no es demostrable por simples principios lógicos, luego no es más que una asociación habitual.

Kant, se opone tanto al racionalismo de Leibniz como al empirismo de Hume, pues ambos sustentan la aritmética en los principios lógicos de la identidad y de la no contradicción. Los juicios regulados por estos principios son denominados "analíticos", no obstante, existe una doble definición de los mismos. La definición positiva de lo analítico se refiere a aquellos que enuncian de modo total o parcial, proposiciones idénticas tales como $a=a$; "el todo es mayor que la parte" 24 . La definición negativa de los enunciados analíticos, por el contrario, es la que regula las operaciones por el principio de no contradicción, como en el caso de reducción al absurdo, que actualmente se considera como el problema de la consistencia.

Según la concepción kantiana, los juicios analíticos por identidad no permiten el incremento del conocimiento, por el contrario, los juicios sintéticos no son demostrables por medios exclusivamente lógicos como en el caso de $a=b$. Kant, observa que las operaciones matemáticas respetan el principio de no contradicción (según lo cual serían analíticas por la definición negativa), pero conllevan una unión de los números que ofrece un nuevo producto como en el ejemplo de $7+5=12$; en la conclusión de la operación aparece un número que

${ }^{21}$ Ibid. p. 90.

${ }^{22}$ Ibid., p. 83.

${ }^{23}$ HUME, David. Resumen del tratado de la naturaleza humana. Madrid: Aguilar, 1973, p. 35.

${ }^{24}$ KANT, Inmanuel. Crítica de la razón pura. Buenos Aires: Losada, 1970. Introducción. p. 158. 
tiene nuevas propiedades, en este caso, es un número par. Si el resultado incrementa el conocimiento entonces es sintético. Los juicios sintéticos pueden ser a priori si se pueden demostrar por medio del entendimiento sin necesidad de recurrir a la experiencia para justificarlos o refutarlos. Si por el contrario, si su verdad requiere de la experiencia para su demostración como cuando se dice que la tierra es redonda, son entonces sintéticos a posteriori. Por esta razón Kant afirma que los postulados geométricos y las deducciones matemáticas son juicios sintéticos a priori, pues incrementan el conocimiento intuitivo formal (puro) del espacio y en el tiempo (sin contenido empírico). En consecuencia la matemática y la geometría, a diferencia de la simple lógica formal clásica, son innovadoras.

Para exponer el conocimiento de la geometría y de la matemática Kant recurre, a sustentarlas en las formas puras de la sensibilidad que son el espacio y el tiempo, en combinación con las categorías de la lógica formal (cantidad, cualidad, relación y modo). De este modo, los postulados de la geometría euclidiana y los principios de la física newtoniana pasan ser esquemas a priori del entendimiento y de la imaginación formal. Tales son los casos de las leyes de la inercia, de acción y reacción, la conservación de la materia; así como en geometría la distancia más corta entre dos puntos es la línea recta, etc. ${ }^{25}$

Las geometrías no euclidianas aunque se fueron imponiendo poco a poco entre los geómetras, encontraron una difícil aceptación por parte de pensadores como Frege, Dühring y Renouvier. Frege, seguidor de la concepción kantiana de la geometría, aceptaba que los axiomas se captan por una intuición pura del espacio que coinciden con los de Euclides. Frege conocía la geometría no euclidiana, pero paradójicamente, era el fundador del programa logicista que trataba de reducir la matemática a la lógica, y, sin embargo, excluye por completo la geometría de su programa. Frege quien se encontraba en completa oposición a la posibilidad de que pudiera haber varias geometrías. El estaba absolutamente convencido de que la única geometría verdadera era la geometría de Euclides, por lo tanto, que las geometrías no euclídeas eran falsas. Más aún, Frege fue un implacable crítico de la concepción kantiana de la aritmética, no obstante, se permitió aceptar sin más y como definitiva la concepción kantiana de la geometría. $^{26}$

${ }^{25}$ Ibid, p. 158-159.

${ }^{26}$ MOSTERINI, Jesús Conceptos y teorías de la ciencia. Madrid, Alianza, 1984, p. 119. 
Para entender las consecuencias pedagógicas y epistemológicas del hecho científico que llevó a las geometrías no euclidianas ayuda bastante la obra del filósofo y matemático francés, Henri Poincaré (1854-1912), estudioso y crítico de la ciencia, quien, en La ciencia y la hipótesis, obra publicada y complementada más tarde con El valor de la ciencia y con la obra Ciencia y método, defenderá como tesis principales: el carácter convencional, intuitivo, constructivo de la ciencia y la libertad creadora del espíritu. Según Poincaré, el científico no se limita a traducir la experiencia sino que él la genera, la corrige y la reconstruye. La reflexión de Poincaré está motivada principalmente por el fulgurante desarrollo de la geometría en el siglo XIX, desarrollo que como se ha visto logró romper con uno de los paradigmas más fuertes de la ciencia matemática desde la época de los griegos, el de la aceptación de la geometría de Euclides como la única geometría valida. Así mismo, en filosofía, las geometrías alternativas, ponen en crisis tanto la "intuición autoevidente", como la reducción de las ciencias formales a los juicios analíticos.

El estudio de las diferentes geometrías posibles desarrolladas durante el siglo XIX llevó a Poincaré a plantearse esta cuestión: puesto que muchas geometrías son posibles, ¿La geometría de Euclides no será solamente una geometría provisional? Y, ¿no podrá también la geometría de Lobachevsky y Riemann convertirse en geometrías que se pueden aplicar como la de Euclides? Para resolver todas estas preguntas Poincaré lleva su cuestionamiento a la naturaleza de los postulados ¿Son acaso los postulados de la geometría juicios sintéticos a priori, como lo sostenía Kant? Para Kant tales juicios son considerados por nuestro entendimiento como necesariamente válidos, sin necesidad de recurrir a la experiencia, sin embargo, incrementan el conocimiento. Los postulados se imponían con tal fuerza al espíritu debido a su propia constitución, que resultaba imposible afirmar lo contrario. Si esto fuese así, es lógico que no pudiera haber geometrías no euclidianas.

Los postulados de la geometría ¿serán, por el contrario, verdades experimentales? ¿Pero es acaso posible llamar "experiencia" al trabajo hecho sobre las relaciones con rectas, puntos, circunferencias formadas con conceptos ideales? Además, argumenta Poincaré, si la geometría fuera una ciencia experimental estaría sometida a una continua revisión de la experiencia. En

${ }^{27}$ POINCARÉ, H. "Naturaleza del razonamiento matemático”. En: La filosofía de la ciencia. Op. Cit., p. 220. 
contra de Leibniz, Poincaré sostiene que la demostración de la aritmética de modo puramente analítico por identidad, sería redundante: "Resulta estéril porque la conclusión no es más que la traducción de las premisas a otro lenguaje" 27 . De otra parte, en la matemática y la geometría, piensa Poincaré: "No se puede demostrar todo y no se puede definir todo, será siempre necesario pedir prestado a la intuición." ${ }^{28}$ En consecuencia para Poincaré:

Los axiomas geométricos no son, por tanto, ni juicios sintéticos a priori ni hechos experimentales.

Son convenciones: nuestra elección entre todas las convenciones posibles es guida por los hechos experimentales, pero permanece libre, y sólo responde a la necesidad de evitar toda contradicción. Por ello, es que los postulados pueden permanecer rigurosamente válidos, aun cuando las leyes experimentales que han determinado su adopción sólo sean aproximadas [...]. Entonces, ¿qué se debe pensar de esta pregunta? ¿Es verdadera la geometría euclidiana?

La pregunta carece de sentido.

Es lo mismo que preguntar si el sistema métrico es verdadero y las antiguas medidas falsas. Una geometría no puede ser más verdadera que otra; solamente puede ser más cómoda. ${ }^{29}$

Otra reflexión importante en la comprensión del desarrollo de las geometrías no euclidianas es el de la invención. ¿Cómo se hacen las matemáticas? ¿Qué es de hecho una invención matemática? Para Poincaré "Es preciso aceptar entonces que el razonamiento matemático tiene por sí mismo una especie de virtud creadora y por consiguiente se distingue del silogismo" 30 . Pero este punto de vista no consideraba la creación en la lógica simbólica contemporánea y sólo juzgaba la lógica formal aristotélica.

La matemática como la geometría se forman mediante una combinatoria de relaciones que constituye una verdadera construcción y es por este incremento del conocimiento que es posible la invención. El razonamiento matemático también incluye la retroacción de los teoremas sobre sus definiciones y axiomas, un retorno del todo a las partes. La construcción implica un “orden”, una unidad

${ }^{28}$ POINCARÉ, H. “Las definiciones matemáticas y la enseñanza”. En: La filosofía de la ciencia. Op. Cit., p. 204.

${ }^{29}$ POINCARÉ, Henri. "Geometrías no euclidianas”. En: Filosofía de la ciencia. México: Universidad Autónoma de México. 1964, p. 160.

${ }^{30}$ POINCARÉ, H. "Naturaleza del razonamiento matemático”. En: La filosofía de la ciencia. Op. Cit., 218-219. 
del conjunto y no una simple yuxtaposición. Este procedimiento que no se muestra en las deducciones, aparece, sin embargo, en los nuevos planteamientos matemáticos. De este modo:

Los matemáticos proceden por "construcción"; construyen combinaciones cada vez más complicadas. Volviendo enseguida, mediante el análisis de esas combinaciones, de esos conjuntos por decirlo así, a sus elementos primitivos, perciben las relaciones de esos elementos y deducen las relaciones de los conjuntos $\operatorname{mismos}^{31}$.

Al parecer las discusiones sobre los fundamentos de la matemática, en gran parte dependen de la definición de ciertos criterios y desde el punto de vista con el que se examinen los problemas, pues algunas veces, los términos empleados, poseen diversos significados o apuntan a diferentes interrogantes. Para Kant, como también para Poincaré las matemáticas son innovadoras, y por esta misma razón, no pueden ser exclusivamente analíticas, es decir, no pueden estar constituidas únicamente por los principios de identidad y no contradicción.

Pero, sería necesario aclarar las tesis de Kant y Poincaré: si atendemos a la definición negativa de los enunciados analíticos, según la cual todos los enunciados lógico-matemáticos deben estar regulados por el principio de no contradicción, todas las ciencias formales serían analíticas desde el punto de vista de la prueba de su consistencia, no obstante, son creativas como dice Poincaré, o novedosas como propone Kant. Esto es posible porque la analiticidad negativa de las deducciones, no es contradictoria con la novedad, pues, el principio de no contradicción, sólo limita: prohíbe la contradicción, no la combinatoria ni las convenciones formales. Deja un gran campo abierto a las conexiones creativas, a las relaciones insólitas.

De un modo similar el término "sintético" aplicado a los enunciados pude adquirir dos significados: a) como enunciados cuya validez no depende de los principios lógicos; en este sentido, las ciencias lógico-matemáticas no podrían estar constituidas simplemente por enunciados sintéticos, pero sus postulados sí podrían ser sintéticos desde el punto de vista de la significación formal, como de hecho aparece en las geometrías alternativas; la aceptación de uno u

${ }^{31}$ Ibid., p. 232. 
otro postulado es una convención formal que se encuentra articulada al conjunto de las definiciones y deducciones, no es arbitraria ni absolutamente libre; y, b) lo "sintético" se puede definir como resultados deductivos que no se encuentran en las premisas sino que dan lugar a las innovaciones; o bien, como enunciados que no expresan exclusivamente principios lógicos aunque están regulados por los mismos. Considerando ésta última definición de enunciado sintético como novedad y, contando con la definición de los enunciados analíticos negativos, la matemática estaría constituida por enunciados mixtos analíticossintético de acuerdo a la perspectiva o, si se prefiere, sus enunciados serían analíticos-constructivos a priori por más que les pese, como decía Kapler, al "griterío de los torpes".

Una situación similar aparece con la definición de "a priori": a) la primera, es la definición de los enunciados formales a priori que son aquellos cuya validez no depende de la experiencia sino de definiciones, axiomas, postulados y deducciones lógico-matemáticas; estos enunciados ni se confirman ni se refutan mediante la observación; en este significado todas las ciencias lógicomatemáticas son necesariamente a priori e incluyen, también, los enunciados analíticos y las convenciones formales; b) la segunda, es la definición del $a$ priori constitutivo que es más amplia y usada en filosofía, se refiere a aquellos enunciados que no sólo son relativamente independientes de la experiencia sino que también son supuestos o "enmarcan" todas las experiencias: todas las experiencia los "mostrarían" pero algunas teorías podrían relativizarlos y reubicarlos como condiciones particulares. Este último significado caracteriza propiamente la filosofía de Kant cuando intenta establecer las condiciones de la experiencia posible (postulados de la geometría euclidiana y principios supuestos por la física clásica) no obstante, también este punto de vista se ha relativizado al igual que la mayoría de las "intuiciones"32 con las geometrías alternativas y la física contemporánea, por un lado $\mathrm{y}$, con la sicogénesis cognitiva, del otro.

${ }^{32}$ Existen diversas escuelas intuicionistas, una de ellas, la de Brouwer, tiene consecuencias en la limitación de algunos teoremas como el de los transfinitos de Cantor, dado que no pueden ser "construidos" por el sujeto: están más allá de su alcance. Cfr. BARKER, S. Filosofía de la matemática. México: U.T.H.A, 1965, pp. 112-120.

Para Poincaré, además de los principios analíticos, hay intuiciones en los puntos de partida, convenciones y construcciones. En Piaget, por el contrario, no hay intuiciones autoevidentes sino como resultado del proceso de construcción: se acepta las matemáticas al infinito y la ampliación de estructuras posibles como las geometrías alternativas. 
Reflexionando sobre el tema de la invención nos dice James Newman en Sigma el mundo de las matemáticas citando a Poincaré, que la invención matemática no consiste sólo en hacer nuevas combinaciones con entes matemáticos ya existentes, lo cual podría hacerlo cualquiera, sino que consiste, fundamentalmente, en discriminar y escoger, pero de modo especial, el inventor en matemáticas es aquél que es capaz de encontrar relaciones insospechadas entre elaboraciones ya conocidas hace tiempo, pero que se consideraban como independientes. Es posible que el inventor tenga ciertos momentos de iluminación súbita, a veces hasta inconsciente, pero lo cierto es que el inventor estaba buscando algo y que una vez pasado el momento de la iluminación debe dedicar mucho tiempo al trabajo consciente, a dar forma a los resultados de la inspiración, a deducir las consecuencias, redactar las demostraciones, y sobretodo, a comprobarlas porque, generalmente, el momento de inspiración da certezas que muchas veces, el trabajo consciente y metódico de reflexión, y comprobación, obliga a desechar. ${ }^{33}$

La reflexión sobre la invención lleva a la creatividad. En el desarrollo de las geometrías no euclidianas hubo matemáticos altamente creativos, ¿pero, cómo debe entenderse la creatividad? El gran químico y pedagogo norteamericano J.J. Lagowski, distingue cuatro etapas en una experiencia creativa: preparación, incubación, iluminación y comprobación (verificación) aclarando que la naturaleza y extensión de cada etapa pueden variar considerablemente de un caso a otro. La creatividad es difícil de reconocer y es difícil prever un acontecimiento creativo, pero una vez ocurrido, las personas entendidas lo reconocerán como tal, como el resultado de un trabajo realmente original, porque la novedad y la originalidad son las características fundamentales del pensamiento creativo que no puede medirse en términos operacionales. ${ }^{34}$

La preparación, o etapa inicial, incluye el tiempo y el esfuerzo gastado en adquirir mucha información y experiencia con el fin de estar listo para llegar a un nuevo punto de vista. El investigador está buscando, quiere encontrar una nueva perspectiva o una nueva solución para un problema. Esta es una etapa de intenso trabajo investigativo, reflexivo y analítico.

33 NEWMAN, John Von Sigma. El mundo de las matemáticas. Barcelona, Grijalbo, tomos V, 1983.

${ }^{34}$ LAGOWSKI, J.J. Página editorial de la revista estadounidense Journal of Chemical, número 5, volumen 62, (mayo 1985). 
Realizado este trabajo previo comienza la incubación, segundo período del proceso creativo, donde no son los procesos lógicos los que llevan necesariamente a la solución creativa como bien lo señalara en alguna ocasión Einstein; a veces la confianza en los poderes de la razón puede empujar al investigador a una solución rápida pero equivocada. Como lo había planteado Poincaré, Lagowski insiste en que el subconsciente es el asiento del pensamiento creativo, porque en él, el investigador no está limitado por las restricciones corrientes y literales del lenguaje consciente y es allí donde el simbolismo es más alegórico y metafórico. Subraya que en dicho nivel la mente puede movilizar con rapidez grandes cantidades de datos y suponer asuntos disímiles en nuevos modelos preceptúales y conceptuales.

La iluminación, o tercera etapa del pensamiento creativo es el momento en el cual el nuevo concepto, o las nuevas posibilidades, irrumpen en lo consciente. Cuando sobreviene la iluminación hay una exaltación de la tensión, una excitación o reacción descrita por muchos como la réplica del jajá!, el jeureka! de Arquímedes cuando descubrió su famoso principio.

El estudio del desarrollo de las geometrías no euclidianas, como el de cualquier otro descubrimiento en ciencia, deja ver que los resultados científicos innovadores son fruto de un gran trabajo investigativo, analítico, crítico y constructivo donde, si bien puede haber jugado mucho el genio inventivo de sus creadores, no puede desconocerse el trabajo histórico de búsqueda de nuevas soluciones que durante siglos inquietó a las mentes más lúcidas de lógicos, y matemáticos, desde la época de los griegos.

\section{Conclusión: origen de las primeras nociones matemáticas y sus repercusiones pedagógicas}

En el siglo XX las matemáticas han logrado un alto grado de abstracción y sofisticación, sin embargo, es claro que la matemática apareció originariamente como parte de la vida diaria del hombre, la supervivencia de la especie humana está relacionada, en gran parte con el desarrollo de los conceptos matemáticos. Los conceptos de número, magnitud y forma se remontan a los primeros días de la raza humana, e incluso se considera que la capacidad de distinguir entre número, tamaño, orden y forma, no son propiedad exclusiva del hombre, sino también de algunos animales, como lo señaló Darwin en El origen del Hombre. ${ }^{35}$

${ }^{35}$ DARWIN, C. El origen del hombre. Medellin: tomado de Editorial Anteo, s.f. 
Es posible que el hombre haya necesitado miles de años para extraer conceptos abstractos de situaciones concretas repetidas. Algunos, por ejemplo, afirman que las matemáticas aparecieron para resolver problemas prácticos, pero, además ciertos estudios antropológicos sugieren que el arte de contar pudo haber aparecido en conexión con rituales religiosos. Las relaciones espaciales, no tiene porque haber tenido exclusivamente una motivación práctica, sino posiblemente estética, para disfrutar de la belleza de la forma, como le pasa a muchos matemáticos modernos. También Aristóteles (384-322 a. J.C.) sitúa los orígenes de la geometría en Egipto, pero pensaba que ella había nacido allí gracias a la amplia clase sacerdotal ociosa.

El origen de la geometría que es sin duda más antigua que el arte de la escritura. Herodoto (historiador griego, llamado el padre de la historia, ( $i 484-i 420$ ? a. de J.C.) por ejemplo, pensaba que la geometría había nacido en Egipto, a partir de la necesidad práctica de volver a medir las tierras después de las inundaciones del río Nilo.

Piaget realiza una comparación entre la historia de la matemática y el aprendizaje inteligente por parte del niño. ${ }^{36}$ Para Piaget, las ciencias lógico-matemáticas tienen su origen en la coordinación de la acción como en los casos de clasificar, formar conjuntos, series de menor y mayor, incluir, dividir, clasificar, reunir, establecer correspondencias, etc. Ciertos empirismos fracasaron al intentar explicar el origen sicológico por medio de la experiencia, pues lo que se abstrae en este caso, es la información sobre propiedades físicas como colores, texturas, pesos, etc. El aprendizaje lógico-matemático, por el contrario, abstrae esquemas de la acción que no incluyen propiedades del objeto, antes bien tratan de nuestra interacción con ellos, como había propuesto la filosofía dialéctica de la praxis al sustentar el desarrollo del entendimiento sobre el trabajo (Hegel, Marx, Engels).

En cuantos a los orígenes Piaget se interesa por investigar el aprendizaje de la geometría a partir de los “conjuntos prácticos", según lo había propuesto Poincaré, pues estos conjuntos corresponden a las formas más elementales de la coordinación sicológica de la acción. En los conjuntos prácticos se da la posibilidad de coordinar movimientos como los del punto de partida y retorno, realizar la operación inversa en la acción, la elección de diversos caminos hacia la misma meta (rodeos),

${ }^{36}$ Cfr. PIAGET, J. Introducción a la epistemología genética: 1 El pensamiento matemático. Buenos Aires, Paidos: 1975, p. 297. 
acumulación de procesos en el movimiento en el espacio y la repetición. Estas operaciones motrices, se abstraen, en múltiples operaciones como la sumatoria, la identidad, la asociatividad, la seriación, las figuras, la operación inversa, etc. Piaget, estudia la génesis de la geometría en el niño en la cual trata de la construcción de conceptos espaciales, topológicos, proyectivos y euclidianos. ${ }^{37}$

Todos los pueblos de la tierra utilizan operaciones lógico-matemáticas primero en forma concreta, en la cual las operaciones se realizan con los objetos, como en los casos de la agrimensura, la construcción de viviendas, la organización en procesos de caza y pesca, ${ }^{38}$ la recolección que organizan topológicamente los espacios, la distribución de bienes que conlleva una cuantificación y asociación de operaciones, etc. Para el caso, no interesa que tales actividades se encuentren asociadas con mitos, ritos o al arte, de igual manera exigen operaciones, primero con los objetos, en concreto y, posteriormente, en abstracto, es decir, en operaciones formales.

Según Piaget, a partir de las operaciones formales, las construcciones axiomáticas de las geometrías alternativas con sus conceptos, postulados, axiomas y deducciones, se separan de la interacción directa de la experiencia con los objetos físicos. Las operaciones formales conducen a generalizaciones, abstracciones y relaciones que sobrepasan la experiencia, como en el caso de la extensión al infinito, de tal manera que las coordinaciones de la geometría clásica (postulado de Euclides, axiomas de Arquímedes) se convierten en un caso especial de las coordinaciones lógicomatemáticas posibles. ${ }^{39} \mathrm{Al}$ mismo tiempo que los nuevos formalismos se alejan de las operaciones concretas, se abren nuevas compuertas, nuevas posibilidades para entender dimensiones del cosmos que sobrepasan nuestros organismos. Así

${ }^{37}$ Cfr. PIAGET, J. y Otros. La epistemología del espacio. Buenos Aires, Ateneo, 1971. Cfr. FLAVELL, J. H. La sicología evolutiva de Jean Piaget. Barcelona: Paidos, 1982.

${ }^{38}$ Los indígenas del Amazonas pescan con flechas, lo cual significa que en términos prácticos tienen que corregir la refracción de la luz y apuntar al pez donde realmente está y no como lo muestra la apariencia.

39 También Gaston Bachelard, al referirse al nuevo espíritu científico que surge a finales del siglo XIX con el nacimiento de las geometrías no euclidianas subraya la importancia que tuvo el entender la geometría como expresión de relaciones, las cuales pueden ser válidas siempre y cuando el desarrollo de cada una de ellas guarde coherencia interna con los postulados y axiomas que se hayan tomado como punto de partida, veamos: "Como se sabe, la equivalencia de las diversas imágenes geométricas ha sido definitivamente establecida cuando se encontró que, unas y otras, correspondían a una misma forma algebraica. Una vez fijada esta correspondencia, ya no era de temerse una contradicción en el sistema de Lobachevsky, o en el de Euclides, puesto que una contradicción geométrica, 
en la astrofísica y la microfísica se vuelve a encontrar el objeto, pero a un nuevo nivel de profundidad al que sólo alcanza nuestro entendimiento axiomáticoexperimental como el planteado por la teoría de la relatividad general de Einstein, aplicando la geometría de Riemann.

Con los estudios de la sicología cognitiva, Piaget retoma las tesis kantianas de la formación del entendimiento, pero lo hace desde el punto de vista genéticoestructural, es decir, de la coordinación y unidad de las operaciones que se van constituyendo en un proceso histórico y en el desarrollo ontogenético individual. Éste punto de vista epistemológico se denomina constructivismo. Desde la perspectiva de la construcción y la reflexión que retorna a sus conceptos y principios primitivos, no existe la intuición como autoevidencia por la constitución del entendimiento como lo suponían Leibniz y Kant, toda intuición, así como toda convención formal es el resultado de la abstracción, la construcción y la reflexión retroactiva. Tampoco sería posible la simple reducción del conocimiento a enunciados analíticos o a tautologías como lo pretende el empirismo lógico contemporáneo pues no explica la convención, la combinatoria ni la innovación, no obstante, la construcción y la creatividad deben realizarse de modo consistente. Para Piaget, sin embargo, existe una diferencia entre la creatividad literaria o social y la construcción lógico-matemática en razón de los formalismos necesarios que se llevan a cabo en las deducciones.

La unilateralidad del empirismo lógico al reducir las ciencias formales a los enunciados analíticos no impide que las ciencias lógico-matemáticas puedan ser comprendidas en la analiticidad negativa, como un límite, dado que siempre intentan probar su consistencia o su coherencia. ${ }^{40}$ De otra parte, la reducción de la matemática

independientemente de su origen, se recuperaría en la forma algebraica y de ahí, en todas las otras geometrías. En suma, el álgebra aglutina todas las relaciones y sólo las relaciones. Es, en tanto que relaciones, que las diversas geometrías son equivalentes. Es en tanto que relaciones que tienen una realidad; no por referencia a un objeto, a una experiencia o a una imagen de la intuición. Cfr. BACHELARD, Gaston. El nuevo espíritu científico. México, Nueva imagen, 1981, p. 32-33.

${ }^{40}$ Hempel, ilustra la tesis de la analiticidad de las teorías geométricas al afirmar que las deducciones son analíticas y, por lo tanto, con verdad exclusivamente formal (pues no dependen de la información física) pero a condición de que se acepten sus postulados. Esto significa que su tesis de la analiticidad de la geometría sería parcial, pues atendería sólo lo deductivo, no a los postulados ni a la construcción. Cfr: HEMPEL, Carl. "Geometría y ciencia empírica.” En: NEWMAN, J. R. (Antología y notas). Matemática, verdad, realidad. Barcelona, Grijalbo, 1974, pp. 33-48. 
a la analiticidad fracasa en muchos aspectos: de una parte, los postulados deberían ser analíticos y precisamente ésto es lo que se ha refutado con las geometrías no euclidianas; de otra, los enunciados analíticos permiten una circularidad pues se definen los unos por los otros y viceversa. ${ }^{41}$ Las tautologías ${ }^{42}$ en cuanto se conciben como la deducción de acuerdo a la forma lógica, se distinguen de los enunciados analíticos por la significación en el lenguaje y las ciencias formales no pueden prescindir del lenguaje cotidiano en sus definiciones. ${ }^{43}$ Además, existen serias dudas de que la inducción matemática de las propiedades de los número al infinito $(n+1)$ pueda ser comprendida como simplemente analítica ${ }^{44} \mathrm{y}$, por último, según el teorema de Gödel ${ }^{45}$ (emparentado con la teoría de los tipos) ningún sistema puede demostrar su propia no contradicción e implica un sistema más fuerte: las demostraciones consistentes deben ser incompletas. ${ }^{46}$ Entonces, las ciencias lógicomatemáticas serían meta-analíticas negativas y meta-constructivas. No podrían demostrar todo, ni construirlo todo.

Otra conclusión importante sobre las consecuencias epistemológicas y pedagógicas del desarrollo de las geometrías no euclidianas, hace referencia a la

${ }^{41}$ Ejemplo de tal circularidad es la definición: "los solteros son no casados". Quine además dice que esto es relativo al significado de "soltero" que puede ser: sin pareja, en unión libre, etc. Cfr. QUINE, W. V. Desde el punto de vista lógico. Barcelona, Ariel, 1962, p p. 50-81.

${ }^{42}$ El punto de vista de la reducción de la lógica a tautologías podría definirse así: "Todas las proposiciones de la lógica, dicen lo mismo. Es decir, nada." WITGENSTEIN, L. Tractatus Logico-Philosophicus. 5.451, Madrid, Alianza, 1973, p. 133.

Piaget por el contrario, plantea que las tautologías no son necesariamente igualación de identidades sino deducciones que transmiten la verdad de los enunciados como en el caso (p. q) (p q). Cfr, PIAGET. Lógica y conocimiento científico. Naturaleza y métodos de la epistemología. Trad. Hugo Acevedo. Buenos Aires, Proteo, 1970, p. 97.

${ }^{43}$ En la geometría existen términos no definidos y los definidos, lo cual constituye una semántica. A partir de los contextos semánticos se definen algunos de sus postulados, como el de la línea recta, a diferencia de las teorías físicas cuyo relevancia involucra también los experimentos. Cfr. BARKER, S. Filosofía de la matemática. Op. Cit., p. 63-68.

${ }^{44}$ Cfr. PAP, A. Teoría analítica del conocimiento. Madrid: Tecnos, 1964. Cap. VI.

${ }^{45}$ Para Quine, el teorema de Gödel refuerza en cierto sentido la concepción intuicionista, pues no puede haber procedimientos completos de la teoría de los números. En ciencias formales por constructivismo, como una variante del intuicionismo, se entiende el punto de vista que exige mostrar cómo se encuentran las conclusiones, si se cumplen esas exigencias es un logro, pero su aspecto cuestionable es que limita las propuestas, y de otro lado, el constructivismo huye de las paradojas, no intenta solucionarlas. Cfr. QUINE, W.V. Filosofía de la lógica. Madrid: Alianza Universidad, 1973, p. 150.

${ }^{46}$ Cfr. RUSSELL, B. "La lógica matemática y la fundamentación en la teoría de los tipos"En: Lógica y conocimiento. Madrid; Taurus, 1966. 
interrelación permanente entre el mundo de la experiencia sensible activa y productiva, y el mundo del puro razonamiento abstracto en el desarrollo y evolución del pensamiento matemático. Esta conclusión también refuerza el punto de vista pedagógico. Si las ciencias formales pueden ser consideradas como construcciones de conceptos y de relacione abstractas, éstas tienen su origen histórico en el trabajo y, los niños y aprenden a partir de la actividad con los objetos, entonces, también la pedagogía de estas ciencias debe ser activa como había entrevisto Pestalozzi:

\begin{abstract}
Ahora bien, lo que ha enseñado la experiencia es precisamente que esos niños que adquirieron las primeras nociones aritméticas de un modo manual, y con el método que he descrito [en la actividad con los objetos] ofrecían dos grandes ventajas en relación con los otros. En primer lugar, eran perfectamente conscientes no sólo de lo que hacían sino también del motivo por el que lo hacían. Se habían enterado de los principios de los que depende la solución; hacían algo más que servirse mecánicamente de una formula ${ }^{47}$.
\end{abstract}

Este aprendizaje constituye precisamente el centro de la investigación epistemológica de la sicología genética que ha desarrollado Piaget. En consecuencia la pedagogía debe remitir, en principio, a las operaciones empíricas activas para elevar cada vez más la abstracción y la construcción creativa de nuevos teoremas y formalismos.

La conexión de la historia de la geometría en relación con la pedagogía, podría resumirse en un texto de Poincaré:

El fin principal de la duda matemática es desarrollar ciertas facultades del espíritu, y, entre ellas la intuición. Es merced a ella que el mundo matemático permanece en contacto con el mundo real; y cuando las matemáticas puras pudieran prescindir, de ella, sería preciso siempre tener recursos para salvar el abismo que separa el símbolo de la realidad. ${ }^{48}$.

\title{
Bibliografía
}

AGAZZI, Evandro y PALADINO, D. Le geometrie non - euclidienne e $i$ fondamenti della geometria. Milán, Mondarori, 1978.

${ }^{47}$ PESTALOZZI, J. H. Cartas sobre la educación infantil. Madrid: Tecnos, 1966, p. 127.
${ }^{48}$ POINCARÉ, H. “Las definiciones matemáticas y la enseñanza”. Op. Cit., p. 202. 
BARKER, Stephen. Filosofía de las matemáticas .México: UTEHA, 1965.

BACHELARD, Gastón El nuevo espíritu científico. México: Nueva imagen, 1985.

BOLYAI, J. The Science absolute of space. Introducción y traducción del Latin de George Bruce H, Austin, Texas: Ed. Neomon. 1896.

BLANCHE, Robert L'axiomatique. París, PUF, 1959.

BOYER, Carl Historia de las matemáticas. Madrid: Alianza, 1999.

BONOLA, Roberto Sobre la geometría de las paralelas y sobre las geometrías no euclidianas. Buenos Aires: Losada, 1945.

CID, Felip Historia de la ciencia. Barcelona: Planeta, tomo I, 1977.

DARWIN, C. El origen del hombre. Medellin: tomado de Editorial Anteo, s.f.

DE LORENZO, Javier. La filosofía de la matemática de Poincaré. Madrid: Tecnos, 1974.

EINSTEIN - INFELD La física, aventura del pensamiento. Buenos Aires: Losada. 1939.

EUCLIDES. Elementos. Trad. María Luisa Puertas. Madrid: Gredos, 1991.

HUME, David. Resumen del tratado de la naturaleza humana. Madrid: Aguilar, 1973.

KANT, Inmanuel. Crítica de la razón pura. Buenos Aires: Losada, 1970.

LEIBNIZ, Gottfried Wilhelm. Muevo tratado sobre el entendimiento humano: Del conocimiento. Libro IV Madrid: Aguilar, 1972.

LAGOWSKI, J.J. Página editorial de la revista estadounidense Journal of Chemical, número 5, volumen 62, (mayo 1985).

LAKATOS, Imre Historia de la ciencia y sus reconstrucciones racionales. Madrid: Tecnos, 1987.

PESTALOZZI, J. H. Cartas sobre la educación infantil. Madrid: Tecnos, 1966.

NEWMAN, John Von. Sigma. El mundo de las matemáticas. Barcelona, Grijalbo, tomos IV y V, 1983.

NEWMAN, J. R. (Antología y notas). Matemática, verdad, realidad. Barcelona: Grijalbo, 1974.

MOSTERINI, Jesús. Conceptos y teorías de la ciencia. Madrid: Alianza, 1984.

PAP, Arthur. Teoría analítica del conocimiento. Madrid: Tecnos, 1964.

PIAGET, J. y Otros. La epistemología del espacio. Buenos Aires: Ateneo, 1971.

PIAGET, J. Introducción a la epistemología genética: 1 El pensamiento 
matemático. Buenos Aires: Paidos, 1975.

PIAGET. Lógica y conocimiento científico. Naturaleza y métodos de la epistemología. Trad. Hugo Acevedo. Buenos Aires: Proteo, 1970.

PIAGET, J. y BETH, E. W. Relaciones entre la lógica formal y el pensamiento real. Trad. Víctor Sánchez de Zavala, Madrid: Nueva Ciencia, 1a Ed. 1968.

POINCARÉ, Henri. La science et l'hypothèse. Paris: Flammarion, 1968.

POINCARÉ, Henri. Filosofía de la ciencia. México: Universidad Autónoma de México. 1964.

POINCARÉ, Henri. Ciencia y método. Madrid: Espasa-Calpe, 1963.

RIEMANN, G.F.B. Riemanniana selecta. Madrid: Ferreirós, 2000.

REALE Giovanni y ANTISERI, Darío Historia del pensamiento filosófico y científico. Barcelona, Herder, Tomos I y III, 1988.

RUSSELL, B. "La lógica matemática y la fundamentación en la teoría de los tipos"- En: Lógica y conocimiento. Madrid; Taurus, 1966.

VERA, Francisco, (Comp). Científicos griegos. Madrid, Aguilar, tomo I., 1970

WITGENSTEIN, L. Tractatus Logico-Philosophicus. Madrid, Alianza, 1973. 